\title{
A Survey on the Short-term Outcome of Microlumbar Discectomy with General versus Spinal Anesthesia
}

\author{
Mohsen Dashtbani, MD, Mehrdad Mokaram Dori, MD*, Mohammad Hassani, MD, \\ Farzad Omidi-Kashani, MD
}

Departments of Orthopedic Surgery and *Anesthesiology, Imam Reza Hospital, Mashhad University of Medical Sciences, Mashhad, Iran

Background: Surgery on the lower thoracic and lumbosacral spine is possible with both general and spinal anesthesia, but most spine surgeons are reluctant to perform the surgery with spinal anesthesia. We aimed to conduct a survey on the short-term outcome of microlumbar discectomy in the patients who had been treated under general or spinal anesthesia.

Methods: In this prospective study, we performed a survey on 72 patients who underwent microlumbar discectomy under general anesthesia (group A) or spinal anesthesia (group B). Demographic characteristics, American Society of Anesthesiologists physical status, duration of operation, blood loss, and complications were all documented. Preoperative and early postoperative (at the time of discharge) disability and pain were assessed by using Japanese Orthopedic Association (JOA) scoring system and a visual analog scale questionnaire.

Results: The two groups were homogenous preoperatively. The mean intraoperative blood loss was less and the mean operating time was shorter in group $A$ than in group B, but there was no statistically significant difference between groups. The rate of postoperative improvement in JOA score and improvement in pain were similar between groups. Anesthetic complications were unremarkable.

Conclusions: Simple lumbar disc operations in the otherwise healthy patients can be safely performed under either spinal or general anesthesia. Both anesthetic methods led to comparable outcomes with minimal complications.

Keywords: Discectomy, Outcome assessment, General anesthesia, Spinal anesthesia

Lumbar disc surgery is undoubtedly the most common spine surgery throughout the world. Although operations on the lower thoracic and lumbosacral spine are possible with either general or spinal anesthesia, most spine surgeons are reluctant to perform them with spinal anesthesia. ${ }^{1,2)}$ In a prolonged and complicated surgery, a surgeon may prefer general anesthesia to avoid airway

Received March 18, 2019; Accepted April 22, 2019

Correspondence to: Farzad Omidi-Kashani, MD

Department of Orthopedic Surgery, Imam Reza Hospital, Mashhad University of Medical Sciences, Imam Reza Square, Mashhad 9137913316, Iran

Tel: +98-915-514-9248, Fax: +98-513-858-3842

E-mail: omidif@mums.ac.ir compromise; however, in simple lumbar discectomy, general anesthesia may not be feasible. Some of the most commonly quoted but not established advantages of regional anesthesia include a decreased incidence of perioperative cardiovascular events, intraoperative hemorrhage, postoperative hypoxia, or pulmonary complications, better intraoperative neural and skin pressure control, postoperative pain control, and cost-effectiveness. ${ }^{3-8)}$

Despite all the comparative studies on spinal and general anesthesia, there is still controversy on the influence of these two different methods of anesthesia on perioperative outcome of surgery. In this study, we aimed to conduct a survey on the short-term outcome of microlumbar discectomy in the patients with lumbar disc herniation 
Dashtbani et al. Lumbar Discectomy by General versus Spinal Anesthesia

Clinics in Orthopedic Surgery • Vol. 11, No. 4, 2019• www.ecios.org

who had undergone the surgery under general or spinal anesthesia.

\section{METHODS}

After obtaining Institutional Review Board approval from deputy of research, Mashhad University of Medical Sciences (IRB No. research/950673//2396) on December 16, 2017 (local ethical committee's code No. IR. MUMS. fm. REC. 1396.06), we performed a survey on patients surgically treated for single-level lumbar disc herniation preand postoperatively. Informed consent was obtained from all patients preoperatively. The patients were alternately assigned to group A (general anesthesia) or group B (spinal anesthesia). The inclusion criteria were pain refractory to more than 6 weeks of conservative treatment, significant progressive neurologic deficit, and single-level spinal involvement in patients aged 20 to 60 years. Patients were excluded if they insisted on a particular type of anesthesia or had nondiscogenic sciatica, revision surgery, cauda equina syndrome, or unstable spine requiring instrumentation or fusion.

All surgical procedures were carried out by a single surgeon (FOK) and a single anesthesiologist (MMD) with similar surgical and anesthetic techniques. ${ }^{9)}$ Demographic characteristics and American Society of Anesthesiologists (ASA) physical status of the patients were all recorded. ${ }^{10)}$ Intra- and postoperative variables including duration of operation (from skin incision to the last skin suture), blood loss, complications, and patient satisfaction rate were documented. We used Japanese Orthopedic Association (JOA) scoring system and a visual analog scale (VAS) questionnaire to assess pre- and postoperative status regarding disability and pain, respectively. ${ }^{11,12)}$ The JOA scoring system assesses subjective symptoms, clinical signs, restriction of activities of daily living, and urinary bladder function. The final score can range from -9 to +29 . On the basis of the score, postoperative improvement rate was calculated as follows: postoperative improvement $=$ (postoperative score - preoperative score) / ( 29 - preoperative score $) \times 100 \%$. Azimi et al. ${ }^{13)}$ previously assessed and approved reliability and validity of this scoring system for low back disorders in Iranian patients. On the VAS questionnaire, the patients were asked to specifically rank their current pain level from zero (no pain) to ten (the worst experienced pain) on a scaled sheet. At the time of discharge, usually one or 2 days after surgery, postoperative clinical assessments were performed and patients were requested to complete the questionnaire again.

\section{Statistical Analysis}

We used SPSS ver. 16.0 (SPSS Inc., Chicago, IL, USA) for statistical assessment. To compare quantitative variables, we used independent $t$-test or Mann-Whitney test, depending on the normal or abnormal distribution of the data. Chi-square and Fisher exact tests were used to compare the qualitative variables. To compare the change in the scores obtained from pre- and postoperative questionnaires, we used repeated measurement and Friedman tests. In all cases, the significance level was set at $p<0.05$.

\section{RESULTS}

There were 72 patients in the two groups (36 patients in each group). Their preoperative characteristics-age, sex, level of disc herniation, and ASA physical status-were homogeneous (Table 1). The mean JOA score in our patients was $10.5 \pm 3.1$ preoperatively, which was improved to $24.5 \pm 2.4$ postoperatively. Table 2 shows JOA score, VAS pain score, intraoperative blood loss, and mean operating time of both groups before and after surgery in detail. Although group B had less intraoperative blood loss and shorter operating time (from skin incision to the last skin suture), there was no statistically significant difference between groups. The only notable difference was the preoperative VAS pain score that was significantly higher in group A. The difference was completely incidental and not related to our study design. The rate of postoperative

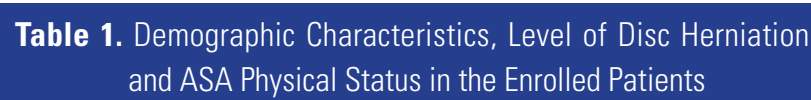

\begin{tabular}{lccc}
\multicolumn{1}{c}{ Variable } & Group A & Group B & $p$-value \\
\hline Age & $46.2 \pm 13.8$ & $43.1 \pm 14.1$ & 0.821 \\
\hline Sex (male:female) & $36(17: 19)$ & $36(20: 16)$ & 0.427 \\
\hline Level of disc herniation & & & 0.325 \\
L4-5 & $16(44)$ & $20(56)$ & \\
L5-S1 & $18(50)$ & $16(44)$ & \\
L3-4 & $2(6)$ & 0 & \\
\hline ASA physical status & & & \\
\hline I & 32 & 28 & \\
\hline II & 4 & 8 & \\
\hline
\end{tabular}

Values are presented as mean \pm standard deviation or number (\%). ASA: American Society of Anesthesiologists, Group A: patients who underwent microlumbar discectomy under general anesthesia, Group $\mathrm{B}$ : patients who underwent microlumbar discectomy under spinal anesthesia. 
Dashtbani et al. Lumbar Discectomy by General versus Spinal Anesthesia

Clinics in Orthopedic Surgery • Vol. 11, No. 4, 2019• www.ecios.org

Table 2. Comparison of Preoperative and Postoperative JOA Score and VAS Score for Pain and Intraoperative Characteristics between the Groups

\begin{tabular}{lccc}
\hline \multicolumn{1}{|c}{ Variable } & Group A & Group B & $p$-value \\
$\begin{array}{l}\text { JOA score } \\
\text { Preoperative }\end{array}$ & & & \\
\hline Postoperative & $24.9 \pm \pm 2.5$ & $24.7 \pm 2.1$ & 0.531 \\
\hline Postoperative improvement (\%) & $73.9 \pm 12.2$ & $75.6 \pm 10.4$ & 0.655 \\
\hline VAS score (leg pain) & & & \\
\hline Preoperative & $8.4 \pm 0.9$ & $6.6 \pm 2.6$ & $0.013^{*}$ \\
\hline Postoperative & $1.0 \pm 0.9$ & $1.4 \pm 1.9$ & 0.436 \\
\hline Postoperative improvement & $7.1 \pm 1.7$ & $5.9 \pm 1.5$ & 0.194 \\
\hline Intraoperative blood loss (mL) & $62.2 \pm 30.6$ & $55.8 \pm 32.6$ & 0.547 \\
\hline Mean operating time (min) & $70.0 \pm 18.1$ & $64.7 \pm 16.1$ & 0.367 \\
\hline
\end{tabular}

Values are presented as mean \pm standard deviation.

JOA: Japanese Orthopedic Association, VAS: visual analog scale, Group $A$ : patients who underwent microlumbar discectomy under general anesthesia, Group B: patients who underwent microlumbar discectomy under spinal anesthesia.

${ }^{*}$ Statistically significant.

improvement in JOA score and improvement in pain were similar between the two groups. Early postoperative complications were trivial: incidental dural tear (one in each group) and superficial wound infection (one in group B).

\section{DISCUSSION}

This was a prospective study comparing effects of the type of anesthesia (spinal versus general) on the outcome of lumbar disc herniation. We enrolled 72 patients with similar preoperative characteristics and assigned them into two groups to compare the results. The type of anesthesia had no effect on intraoperative characteristics and surgical outcome of lumbar discectomy. The spinal anesthesia group had less intraoperative blood loss and shorter operating time, but the difference was not statistically significant.

An overview of the literature suggests that spinal anesthesia has been used for lower thoracic and lumbosacral operations, and it has not yet been replaced by general anesthesia. ${ }^{14-16)}$ In a case-control study on 76 patients undergoing microdiscectomy for lumbar disc herniation (43 patients with spinal and 33 patients with general anesthesia) reported by McLain et al., ${ }^{17)}$ the operating time was longer in the general anesthesia group as in our study. They also found some other benefits of spinal anesthesia: shorter anesthesia time, less urinary retention, less analgesic requirement, less nausea and emesis, and shorter hospital stay-these benefits could be obtained without compromising patient's and surgeon's satisfaction. Thus, they recommended spinal anesthesia at least as an option for anesthesia in relatively small lumbar operations.

In our study, in patients undergoing spinal anesthesia, intraoperative hemorrhage was relatively low, but the difference was not statistically significant. We did not consider hemodynamic characteristics, whereas Becq et al., ${ }^{18)}$ in a study of 83 patients ( 43 spinal and 40 general anesthesia for lumbar discectomy), analyzed hemodynamic indices. They found that the mean arterial pressure and heart rate were higher in the spinal anesthesia group and concluded that this type of anesthesia for simple lumbar discectomy is acceptable hemodynamically. Another study conducted by Attari et al. ${ }^{15)}$ also suggested that the amount of intraoperative hemorrhage in the spinal anesthesia group is relatively lower, which supports the findings of our study. This advantage could be particularly important in elderly patients but because of the enrolling criteria, we could not evaluate the effect of age in our study. ${ }^{16)}$ The shorter duration of operation and less intraoperative blood loss in the patients with spinal anesthesia than in those with general anesthesia have also been demonstrated in a retrospective study of 544 patients with lumbar spine surgery conducted by Pierce et al. ${ }^{19)}$

Tetzlaff et al. ${ }^{20)}$ retrospectively reviewed 803 patients with elective spine surgery (611 spinal and 192 general anesthesia). Nausea, deep vein thrombosis, hypertension, and increased heart rate were more common among the general anesthesia group, whereas mild hypotension and bradycardia were more common in the spinal anesthesia group. In our study, we did not directly investigate the hemodynamic changes during surgery, but the reason for the lower level of intraoperative blood loss in the spinal anesthesia group may have been due to the differences in hemodynamic changes as shown in the study of Tetzlaff et al. ${ }^{20)}$ The authors recommended spinal anesthesia as an effective anesthetic method with a lower rate of adverse events in elective lumbar spine surgery on the basis of comparison with general anesthesia.

Recently, Meng et al., ${ }^{2)}$ in a systematic review and meta-analysis of randomized controlled trials (eight studies with 625 patients), compared the impact of spinal versus general anesthesia in lumbar disc surgery. They could not find significant differences in most perioperative outcome (hemodynamic changes, intraoperative blood loss, surgical time, nausea, vomiting, and analgesic requirement) between the two groups. The results of our study 
Dashtbani et al. Lumbar Discectomy by General versus Spinal Anesthesia

Clinics in Orthopedic Surgery • Vol. 11, No. 4, 2019• www.ecios.org

are in line with these results and there was no significant difference in the clinical outcomes of these two methods of anesthesia.

Our study clearly showed that the improvement in pain and disability at the time of discharge was comparable between the spinal and general anesthesia groups. This indicates that the main determinant of outcome in lumbar spine surgery is the successful deletion of the underlying cause, not the anesthetic method (as pointed out by Meng et al. ${ }^{2)}$.

Although it seems that spinal anesthesia has certain advantages over general anesthesia, this method cannot be recommended for all patients. ${ }^{2,21)}$ Partial or total failure of anesthesia may occur and then obligatory change to general anesthesia becomes necessary although this failure did not occur in our patients. Another disadvantage of spinal anesthesia is its ineffectiveness for protracted surgery, even though some authors believe that spinal anesthesia is safe for operations lasting up to 3.5 hours. ${ }^{16)}$ Since our study was related to simple lumbar disc surgery, none of the operations lasted more than 90 minutes. Other reported trivial complications of this type of anesthesia include transient hypotension (due to the leakage of drug into the epidural space) and postdural puncture headache..$^{22,23)}$ Nowadays, by using an appropriate technique and needle size and design, this last complication has become a very rare event. ${ }^{23)}$

Our study, despite being prospective, have some important flaws. First, we did not record intraoperative hemodynamic changes; therefore, we could not compare the patients anesthetically. Second, satisfaction rate of the surgeon, anesthesiologist, and the patient was not investigated, although it is one of the important criteria for choosing a certain anesthetic type. The last defect in our study is the relatively small study population. This may have to do with a cultural origin. In Iran, from ancient times, lumbar puncture has been considered as an inappropriate, painful, and complicated maneuver. This longheld perception may have played an important role in the number of volunteers participating in the study.

In conclusion, simple lumbar disc surgery in the otherwise healthy patients can be safely performed under either spinal or general anesthesia to achieve comparable outcome with trivial complications. Therefore, we think that final selection of the anesthetic type mostly depends on the operating room conditions, knowledge, convenience, and experience of both anesthesiologists and surgeons.

\section{CONFLICT OF INTEREST}

No potential conflict of interest relevant to this article was reported.

\section{ACKNOWLEDGEMENTS}

The authors thank Student Research Committee, Faculty of Medicine, Mashhad University of Medical Sciences for financial support.

This paper is based on an orthopedic resident's thesis (Mohsen Dashtbani, MD) with ID number of 950673 and regional ethical approval.

\section{REFERENCES}

1. Kahveci K, Doger C, Ornek D, Gokcinar D, Aydemir S, Ozay R. Perioperative outcome and cost-effectiveness of spinal versus general anesthesia for lumbar spine surgery. Neurol Neurochir Pol. 2014;48(3):167-73.

2. Meng T, Zhong Z, Meng L. Impact of spinal anaesthesia vs. general anaesthesia on peri-operative outcome in lumbar spine surgery: a systematic review and meta-analysis of randomised, controlled trials. Anaesthesia. 2017;72(3):391-401.

3. Modig J, Karlstrom G. Intra- and post-operative blood loss and haemodynamics in total hip replacement when performed under lumbar epidural versus general anaesthesia. Eur J Anaesthesiol. 1987;4(5):345-55.

4. Rodgers A, Walker N, Schug S, et al. Reduction of postoperative mortality and morbidity with epidural or spinal anaesthesia: results from overview of randomised trials. BMJ.
2000;321(7275):1493

5. Urwin SC, Parker MJ, Griffiths R. General versus regional anaesthesia for hip fracture surgery: a meta-analysis of randomized trials. Br J Anaesth. 2000;84(4):450-5.

6. Indelli PF, Grant SA, Nielsen K, Vail TP. Regional anesthesia in hip surgery. Clin Orthop Relat Res. 2005;441:250-5.

7. Sakura S. Epidural anesthesia and spinal anesthesia in the elderly. Masui. 2007;56(2):130-8.

8. Scott NB, Kehlet H. Regional anaesthesia and surgical morbidity. Br J Surg. 1988;75(4):299-304.

9. Williams RW. Microlumbar discectomy: a conservative surgical approach to the virgin herniated lumbar disc. Spine (Phila Pa 1976). 1978;3(2):175-82.

10. Mayhew D, Mendonca V, Murthy BVS. A review of ASA 
Dashtbani et al. Lumbar Discectomy by General versus Spinal Anesthesia

Clinics in Orthopedic Surgery • Vol. 11, No. 4, $2019 \bullet$ www.ecios.org

physical status: historical perspectives and modern developments. Anaesthesia. 2019;74(3):373-9.

11. Fukui M, Chiba K, Kawakami M, et al. JOA Back Pain Evaluation Questionnaire (JOABPEQ)/JOA Cervical Myelopathy Evaluation Questionnaire (JOACMEQ). The report on the development of revised versions. April 16, 2007. The Subcommittee of the Clinical Outcome Committee of the Japanese Orthopaedic Association on Low Back Pain and Cervical Myelopathy Evaluation. J Orthop Sci. 2009;14(3):348-65.

12. Wewers ME, Lowe NK. A critical review of visual analogue scales in the measurement of clinical phenomena. Res Nurs Health. 1990;13(4):227-36.

13. Azimi P, Shahzadi S, Montazeri A. The Japanese Orthopedic Association Back Pain Evaluation Questionnaire (JOABPEQ) for low back disorders: a validation study from Iran. J Orthop Sci. 2012;17(5):521-5.

14. Silver DJ, Dunsmore RH, Dickson CM. Spinal anesthesia for lumbar disc surgery: review of 576 operations. Anesth Analg. 1976;55(4):550-4.

15. Attari MA, Mirhosseini SA, Honarmand A, Safavi MR. Spinal anesthesia versus general anesthesia for elective lumbar spine surgery: a randomized clinical trial. J Res Med Sci. 2011;16(4):524-9.

16. Lessing NL, Edwards CC 2nd, Brown CH 4th, et al. Spinal anesthesia in elderly patients undergoing lumbar spine surgery. Orthopedics. 2017;40:e317-22.
17. McLain RF, Tetzlaff JE, Bell GR, Uwe-Lewandrowski K, Yoon HJ, Rana M. Microdiscectomy: spinal anesthesia offers optimal results in general patient population. J Surg Orthop Adv. 2007;16(1):5-11.

18. Becq MC, Verdin M, Riegel B, et al. Hemodynamic effects of genu-pectoral position during the surgery of lumbar disk herniation: spinal anesthesia versus general anesthesia. Agressologie. 1994;34 Spec No 1:49-50.

19. Pierce JT, Kositratna G, Attiah MA, et al. Efficiency of spinal anesthesia versus general anesthesia for lumbar spinal surgery: a retrospective analysis of 544 patients. Local Reg Anesth. 2017;10:91-8.

20. Tetzlaff JE, Dilger JA, Kodsy M, al-Bataineh J, Yoon HJ, Bell GR. Spinal anesthesia for elective lumbar spine surgery. J Clin Anesth. 1998;10(8):666-9.

21. Hassi N, Badaoui R, Cagny-Bellet A, Sifeddine S, Ossart M. Spinal anesthesia for disk herniation and lumbar laminectomy: apropos of 77 cases. Cah Anesthesiol. 1995;43(1):215.

22. Fournet-Fayard A, Malinovsky JM. Post-dural puncture headache and blood-patch: theoretical and practical approach. Ann Fr Anesth Reanim. 2013;32(5):325-38.

23. Campbell DC, Douglas MJ, Pavy TJ, Merrick P, Flanagan ML, McMorland GH. Comparison of the 25-gauge Whitacre with the 24-gauge Sprotte spinal needle for elective caesarean section: cost implications. Can J Anaesth. 1993;40(12):1131-5. 